\title{
Comparative Evaluation of Micronuclei in Exfoliated Urothelial Cells in Patients with Smoking and Smokeless Tobacco-associated Lesions: A Prospective Quantitative Study
}

\author{
1Janaki Subramanian lyer, ${ }^{2}$ Jigna Pathak, ${ }^{3}$ Shilpa Patel, ${ }^{4}$ Leela Poonja, ${ }^{5}$ Niharika Swain
}

\begin{abstract}
Objective: To clinically evaluate various tobacco-associated lesions and to evaluate and compare the micronucleus (MN) assay in exfoliated urothelial cells in patients with smoking and smokeless tobacco-associated lesions.

Materials and methods: This study was conducted in the Department of Oral Pathology and Microbiology, Mahatma Gandhi Mission's Dental College and Hospital, Navi Mumbai, from October 2012 to June 2013. One hundred cases having tobacco habits (smoking or smokeless) and clinically detectable tobacco-associated lesions were included. Exfoliated urothelial cytosmears were prepared, stained with Papanicolaou and slides were scored for $\mathrm{MN}$.
\end{abstract}

Results: All cases $(n=100)$ were found to have tobaccoassociated lesions that were clinically detectable. Voided urine samples were collected from all cases $(n=100)$ who indulged in smoking and smokeless tobacco habit, with males $(n=71)$ and females $(n=29)$, whose ages ranged from 19 to 75 years. We observed that out of the 100 cases evaluated, 12 cases showed the presence of $\mathrm{MN}$ in the urine cytosmear. Of these 12 cases, $n=10$ were bidi smokers and $n=2$ were betel quid chewers. Owing to insufficient population of urothelial cells in the cytosmear, MN evaluation could not be statistically proved.

Conclusion: Although, MN score in the urothelial cells could not be statistically assessed, due to insufficient number of urothelial cells, our observations reveal that MN count seems to be increased in smokers than smokeless tobacco users. Thus, we urge the need for further studies to highlight the comparative evaluation of MN score between smokers and smokeless tobacco on urothelial cells.

Keywords: Cytosmear, Genotoxicity, Micronucleus, Tobacco, Urothelial.

How to cite this article: Iyer JS, Pathak J, Patel S, Poonja L, Swain N. Comparative Evaluation of Micronuclei in Exfoliated Urothelial Cells in Patients with Smoking and Smokeless Tobacco-associated Lesions: A Prospective Quantitative Study. J Contemp Dent 2015;5(2):93-97.

Source of support: Nil

Conflict of interest: None

\footnotetext{
${ }^{1}$ Postgraduate Student, ${ }^{2,4}$ Professor

${ }^{3}$ Professor and Head, ${ }^{5}$ Lecturer

${ }^{1-5}$ Department of Oral Pathology and Microbiology, MGM Dental College and Hospital, Navi Mumbai, Maharashtra, India

Corresponding Author: Janaki Subramanian lyer, Postgraduate Student, Department of Oral Pathology and Microbiology, MGM Dental College and Hospital, Navi Mumbai, Maharashtra, India Phone: 022226365378, e-mail: drjanakisiyer@gmail.com
}

\section{INTRODUCTION}

Oral cancer is the sixth most common cancer worldwide and the most common in India. ${ }^{1}$ Approximately, 70,000 cases and over 48,000 oral cancer-related deaths occur yearly. ${ }^{2}$ Tobacco usage is one of the prime factors for the escalating cancer rates. Epidemiological studies show that the risk of developing oral cancer is 5 to 9 times greater for tobacco consumers than non-consumers. ${ }^{3}$ Tobacco is most commonly smoked as cigarettes, bidis, cigars and loose tobacco in pipes. Smokeless tobacco is usually placed in the oral and nasal cavities against the mucosal sites that permit the absorption of nicotine into the body. In India, tobacco is commercially available in bidi, cigarette, cigar smoking tobacco forms, or gutkha, mawa, mishri, admixed with arecanut and slaked limesmokeless tobacco forms. ${ }^{4,5}$

Benzo[a]pyrene and other polycyclic aromatic carcinogens are the most important carcinogenic agents in smoke, while unburnt tobacco contains 28 carcinogens of which the most harmful are the tobacco specific nitrosamines (TSNAs). The metabolites of nitrosamines, such as carcinogenic TSNAs $\mathrm{N}^{\prime}$-nitrosonornicotine (NNN), 4-(N-methyl-N-nitrosamino)-1-(3-pyridyl)1-butanone (NNK) and $\mathrm{N}$-nitrosoanabasine as well as the volatile nitrosamines $\mathrm{N}$-nitrosodimethylamine and $\mathrm{N}$-nitrododiethylamine, have been detected in saliva of tobacco chewers. ${ }^{4-6}$ Other cancer causing substances include formaldehyde, acetaldehyde, cortonaldehyde, hydrazine, arsenic, nickel, cadmium and benzopyrene. ${ }^{6}$ Tobacco smoke contains more than 60 carcinogenic combustion products. In particular, NNN, NNK and polycyclic aromatic hydrocarbons have been linked with genotoxicity. ${ }^{5}$ Nitrosamines are also produced by the alkaloids in arecanut which are also carcinogenic. ${ }^{7}$ These agents are known to cause adverse effects at a cellular level, either directly at the site of placement or by indirect systemic effects. Various tobacco-associated clinically detectable oral lesions are tobacco keratosis, leukoplakia, oral lichen planus and oral submucous fibrosis. These mucosal changes are well documented to predispose carcinoma. ${ }^{8}$ 
Exfoliative cytology is the study and interpretation of the characteristics of cells that flake off, whether naturally or artificially, from mucosa. ${ }^{9}$ Continuous exfoliation of epithelial cells is a part of physiological turnover. Deeper cells which are strongly adhered in normal conditions become loose in the case of pathology and exfoliate along with the superficial cells. ${ }^{10}$ Thus, the oral epithelial cells are the initial site affected by the carcinogens. The effect of the carcinogen or its metabolites can also be detected in urine, blood, feces, exfoliated cells, sweat, nails, etc. ${ }^{11}$ The normal bladder epithelium, called urothelium, formerly known as transitional epithelium, reveals neoplastic transformation on exposure to mutagens. Exfoliated bladder cells are epithelial cells sloughed from the surface of the genitourinary tract that turn over in the bladder every 1 to 3 weeks. Because epithelial cells are derived from basal cells, recent genetic damage to the basal layer of the bladder could be reflected in the exfoliated urothelial cells. ${ }^{11}$

Reactive oxygen species (ROS) induced by the carcinogens lead to toxic mutagenic changes. Cytogenetic markers, such as chromosome aberration assay, sister chromatid exchange assay and micronucleus (MN) assay have been widely used as an indicator of genotoxic events. Micronuclei is an additional small nucleus situated around the main nucleus formed due to either direct damage to chromosomes (clastogens) or of the spindle apparatus (aneuploidogens/aneugens). ${ }^{12,13}$ It is initiated by various agents, such as radiation therapy, tobacco and alcohol. ${ }^{12,13}$ Nuclear stains, such as Papanicolaou (PAP), Giemsa and Feulgen help as visual aids in MN assay.

Thus, this study was undertaken to assess and compare the MN assay in exfoliated urothelial cells of patients with clinically evaluated smoking and smokeless tobaccoassociated lesions.

\section{MATERIALS AND METHODS}

This study included 100 healthy individuals with the habit of tobacco consumption either of smoking or smokeless form and clinically detectable tobacco-associated lesions retrieved from Department of Oral Pathology and Microbiology. Nonhabit-associated oral lesions, and alcoholics were excluded from this study. Details of these cases were recorded in a case history format to obtain the history of the relevant habits (either smoking or smokeless). The exfoliated urothelial cytosmears involved the centrifugation of voided midstream urine with subsequent suspension of the pellet in phosphate buffered saline. ${ }^{14}$ These alcohol (100\% methanol) fixed smears were stained with PAP and slides were scored for MN.

Cytological interpretation of exfoliated urinary sediments were classified as follows: ${ }^{15}$
- Negative-normal transitional cells.

- Atypia-atypical cells in loose clusters with slightly increased nuclear cytoplasmic ratio with fine chromatin and small nucleoli.

- Suspicious-cells with abnormal features short of a definite diagnosis of malignancy.

- Positive-loose clusters or isolated cells with increased nuclear cytoplasmic ratio with fine to coarse chromatin and prominent nucleoli.

Scoring of MN: Micronucleus in 1,000 well preserved urothelial cells (Figs 1 to 3 ) either single or in groups were counted in exfoliated urothelial cytosmear, in different areas of the smear in a zig zag manner. Clearly defined cells were counted avoiding clumping of folded cells and distorted cells. Smears were observed under 100 and 400x of a binocular light microscope. Criteria laid down by Fenech was used for the identification of $\mathrm{MN}:^{16}$

- Being less than $1 / 3$ and more than $1 / 16$ diameter than the main nucleus.

- Being on the same focus plane.

- Not superimposing to the main nucleus.

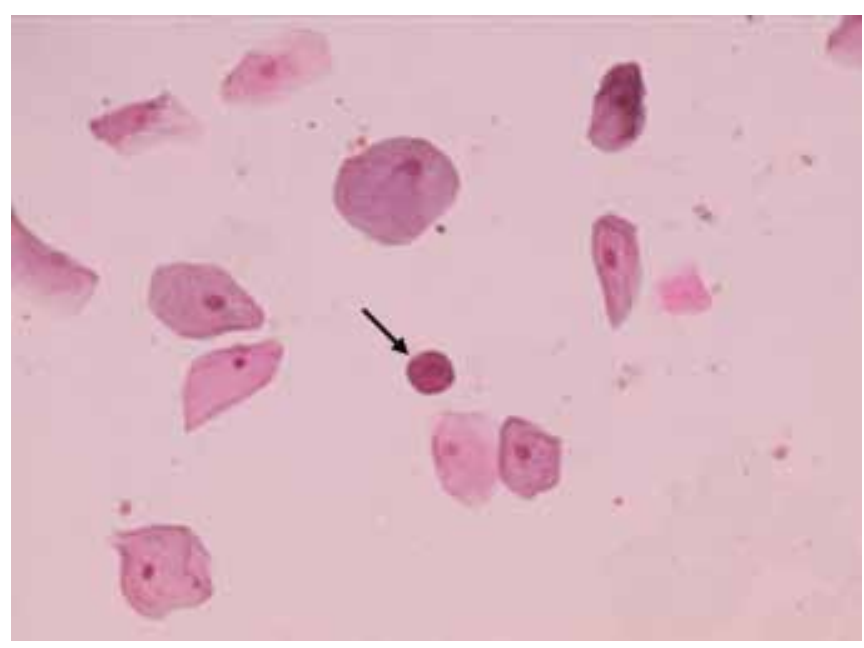

Fig. 1: Cytosmear of voided urine with squamous cell and urothelial cell (arrow) (PAP stain-400x)

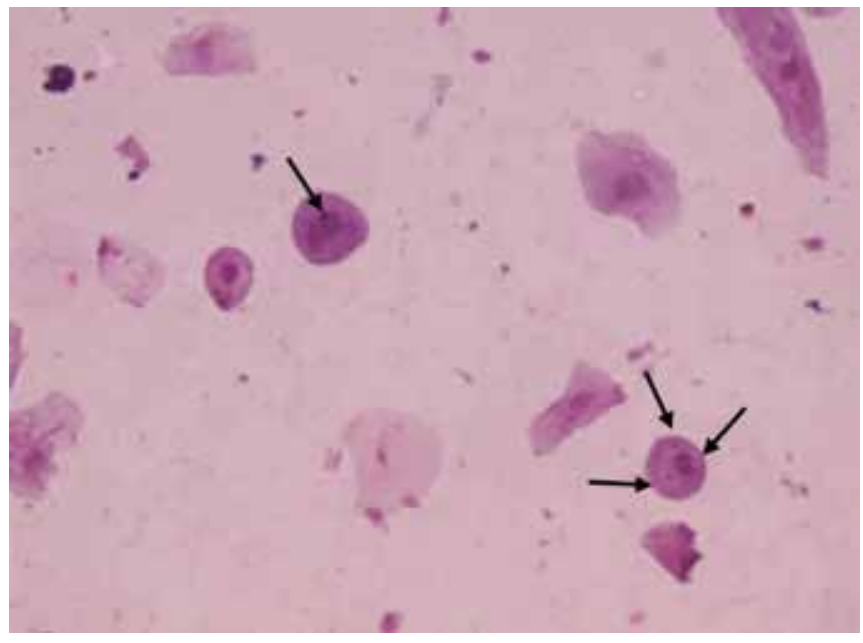

Fig. 2: Urothelial cell with single and multiple MN (arrows) (PAP stain-400x) 


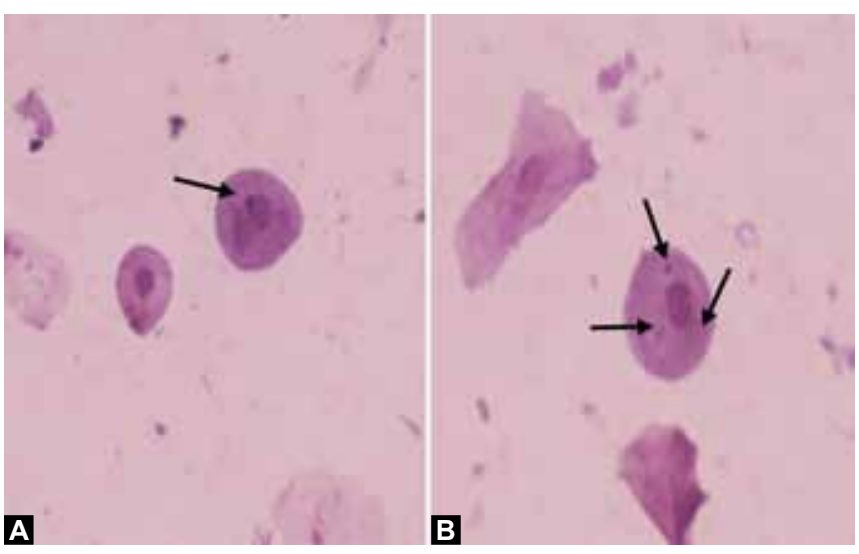

Figs $3 A$ and $B$ : Urothelial cell with single $(A)$ and multiple $M N(B)$ (arrows) (PAP stain)

- Having the same color, texture and refraction as the main nucleus.

- Having a smooth round or oval shape.

- Having limits clearly distinguishable from the main nuclear membrane if touching the main nucleus.

Note: All dead and degenerative cells (karyolysis, karyorrhexis, nuclear fragmentation) were excluded from evaluation. Nuclear blebbing (MN like structure connected with the main nucleus with a bridge) was also not considered.

The slides were seen by two independent observers, and the number of MN was counted in all the urothelial cells.

\section{RESULTS}

\section{Clinical Presentation}

Male patients $(\mathrm{n}=71)$ indulged in tobacco-associated habits and had clinically detected lesions more frequently than female patients $(n=29)$; age of patients ranged from 19 to 75 years. Most patients indulged in tobacco chewing habit $(n=69)$, i.e. gutkha chewers $(n=12)$, betel nut chewers $(n=28)$, betel quid users $(n=19)$, mawa users $(n=2)$ and mishri users $(n=15)$. While all the smokers $(n=31)$ consumed bidi (Graphs 1 and 2). This study evaluated the oral tobacco-associated lesions clinically as oral submucous fibrosis $(n=35)$, leukoplakia $(n=26)$, tobacco pouch keratosis $(n=24)$, lichen planus $(n=15)($ Graph 3$)$.

\section{Cytological Parameters}

Voided urine samples were collected of cases $(n=100)$ who indulged in smoking $(n=31)$ and smokeless $(n=69)$ tobacco habit. We observed that out of the 100 cases evaluated, 12 cases showed the presence of $\mathrm{MN}$ in the urine cytosmear. Among these 12 cases, 10 cases were bidi smokers $(n=10)$ as well as betel quid chewers $(n=2)$. However, owing to insufficient population of urothelial cells in the cytosmear, $\mathrm{MN}$ evaluation could not be statistically proved.

\section{DISCUSSION}

Micronuclei are small chromatin bodies in the cytoplasm that originate from chromosome fragments or whole chromosome that lag behind at Anaphase during nuclear

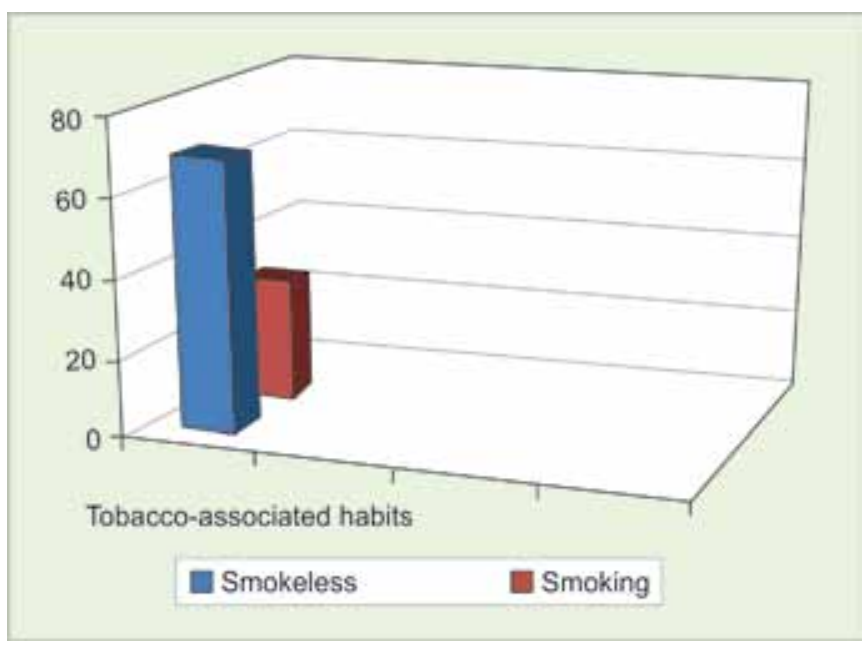

Graph 1: Number of patients having smokeless and smoking habits. The cases with smokeless tobacco habits were significantly higher

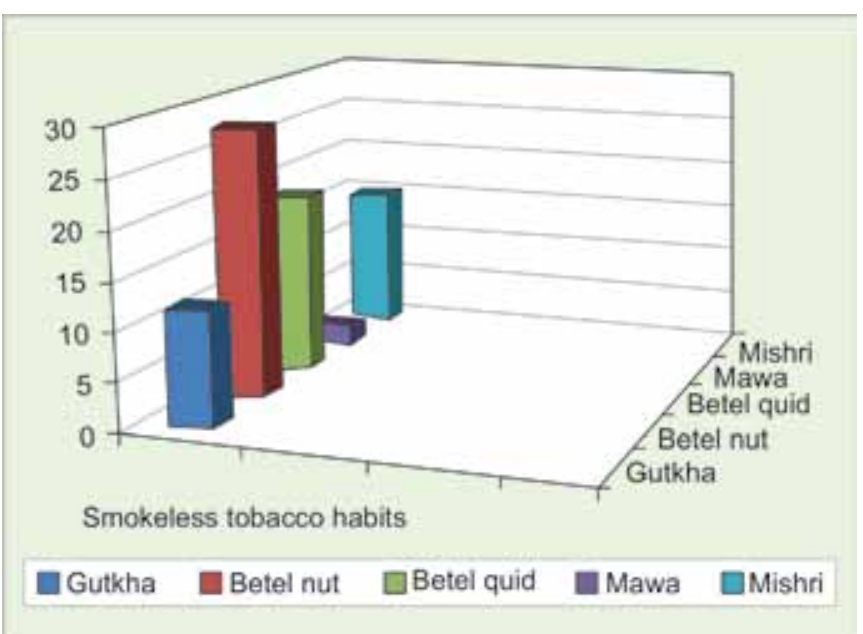

Graph 2: Number of patients with various smokeless tobacco habits

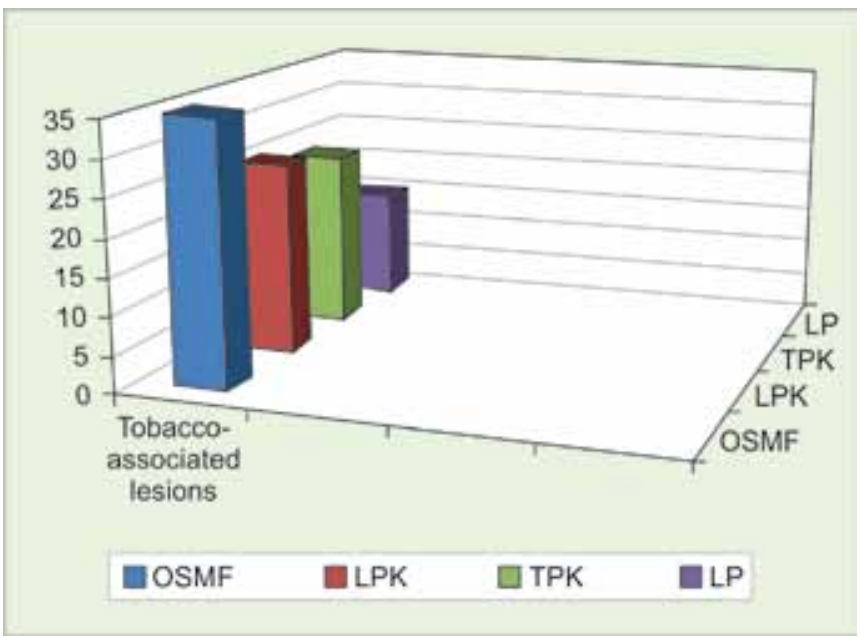

Graph 3: Various tobacco-associated clinically detectable lesions. Oral submucous fibrosis cases were significantly higher 
division. ${ }^{17}$ In humans, MN can be easily assessed in erythrocytes, lymphocytes, and exfoliated epithelial cells (e.g. oral, urothelial, nasal) to obtain a measure of genome damage induced in vivo. The main aim of this prospective study was to evaluate the role of $\mathrm{MN}$ in exfoliated urothelial cells as a potential marker, to determine the genotoxic effects caused by smoking and smokeless tobacco-associated habits.

In our study, on correlating gender with tobacco habits (smoking and smokeless forms) and tobacco-associated lesions, we found, that males ( $\mathrm{n}=71)$ accounted for $71 \%$ and females $(n=29)$ accounted for $29 \%$. Our findings were in agreement with WHO SEARO ${ }^{18}$ and Sarswathi et al. ${ }^{19}$ Relatively increased consumption of tobacco products, either in smoking or smokeless forms among males led to various clinically detectable oral lesions, when compared to female patients.

In our study, the patients who indulged in smokeless tobacco habit $(n=69)$ accounted for $69 \%$ of sample, while smokers $(\mathrm{n}=31)$ accounted for $31 \%$ of the sample. As documented by World Health Organization, South-East Asia Regional Office, ${ }^{18}$ states of Maharashtra and Bihar, predominantly constituted smokeless tobacco users.

Among the smokeless tobacco users $(n=69)$, betel nut chewers ( $\mathrm{n}=28$ ) accounted for $40.57 \%$, betel quid users $(n=19)$ accounted for $27.53 \%$, mishri users $(n=15)$ accounted for $21.73 \%$ gutkha chewers $(n=12)$ accounted for $17.39 \%$, and mawa users ( $\mathrm{n}=2$ ) accounted for $2.89 \%$. Consumption of betel nut and tobacco products has recently increased due to commercially available inexpensive sachets. All smokers $(n=31)$ consumed bidi. In India, bidi smoking is more common than cigarette or any other forms of smoking tobacco due to affordable rate. The carcinogenic metabolites present in smoking and smokeless forms of tobacco induce genotoxic changes at a cellular level due to excessive formation of ROS. ${ }^{7}$

The oral tobacco-associated lesions clinically detected in this study were oral submucous fibrosis $(n=35)$ accounted for $35 \%$, leukoplakia $(n=26)$ that accounted for $26 \%$, tobacco pouch keratosis ( $n=24)$ accounted for $24 \%$ and lichen planus $(n=15)$ accounted for $15 \%$. World Health Organization emphasizes that these potentially malignant disorders are forerunners to cancer development. ${ }^{20}$

The effect of the ingested or inhaled carcinogen or its metabolites can be detected in urine, blood, feces, exfoliated cells, sweat, nails, etc. ${ }^{11}$ The urothelium reveals neoplastic transformation on exposure to mutagens. Exfoliated bladder cells are epithelial cells sloughed from the surface of the genitourinary tract that turn over in the bladder every 1 to 3 weeks. Because epithelial cells are derived from basal cells, recent genetic damage to the basal layer of the bladder could be reflected in the exfoliated cells. ${ }^{11}$ Ribeiro et al, Cid et al, Rosin and Anwar,
Moore et al, Lehucher-Michel et al, Burgaz et al, Murray and Edwards, Fontana et al and Fortin et $\mathrm{al}^{11,14}$ have explored the benefits of performing this test in exfoliated urothelial cells in urine samples as a biological marker of the genotoxic effects of bladder mutagenic agents, like ingested chemical poisoning, tobacco, etc. The present prospective study also evaluated and compared the $\mathrm{MN}$ count in urothelial cells of patients $(n=100)$ with tobacco habits. Voided urine samples were collected from all $(n=100)$ cases, with males $(n=71)$ and females $(n=29)$; who indulged in smoking and smokeless tobacco habit. The cytological interpretation of the exfoliated urine cytosmears were all graded as grade 1 (negative-normal transitional cells) per the criteria laid down by Misra et al. ${ }^{15}$ The MN of 1,000 urothelial cells was scored. Owing to the insufficient population of urothelial cells in the cytosmear, accurate MN score could not be ascertained. Micronuclei evaluation in the urothelial cells of patients with habit and detectable lesions could not be statistically evaluated owing to insufficient population of urothelial cells in the exfoliated urothelial cytosmear. Though we observed the presence of $\mathrm{MN}$ in urothelial cells among only 12 cases on a sampling of 100, it was relatively higher among smokers than smokeless tobacco users, we can concur that the genotoxic effects of the systemic absorption of smoking can be evaluated by MN frequency. Fontana L et $\mathrm{al}^{11}$ and Zamani AG et al. ${ }^{21}$ Twenty-two observed higher mean MN score in urothelial cells of smokers as compared to healthy controls. However due to unsatisfactory number of urothelial cells, the same could not be statistically proven.

\section{CONCLUSION}

A prospective study was performed to evaluate and compare the MN count in exfoliated urothelial cells in patients with smoking and smokeless tobacco-associated lesions. Our observations reveal that $\mathrm{MN}$ count seems to be increased in smokers than smokeless tobacco users, although $\mathrm{MN}$ score in the urothelial cells could not be statistically assessed due to inadequate number of exfoliated cells. Thus, we urge the need for further studies to highlight the comparative evaluation of MN score between smokers and smokeless tobacco users on urothelial cells.

\section{REFERENCES}

1. Warnakulasuriya S. Living with oral cancer: epidemiology with particular reference to prevalence and life-style changes that influence survival. Oral Oncol 2010;46(6):407-410.

2. National Cancer Registry Programme. Three years report of population based cancer registries 2006 to 2008. Time trends in cancer incidence rates: 1982-2005. Bengaluru ICMR: 2009.

3. Neufeld KJ, Peters DH, Rani M, Bonu S, Bronner RK. Regular use of alcohol and tobacco in India and its association with 
age, gender and poverty. Drug Alcohol Dependence 2005; 77(3):283-291.

4. Rodu B, Jansson C. Smokeless tobacco and oral cancer: a review of the risks and determinants. Critical Reviews in Oral Bio Med 2004;15(5):252-263.

5. Proia N, Paszkiewicz GM, Sullivan Nasca MA, Franke GE, Pauly JL. Smoking and smokeless tobacco-associated human buccal cell mutations and their association with oral cancer-a review. Cancer Epidemiology, Biomarkers and Prevention 2006;15(6):1061-1077.

6. Raveendran R, Nath SG. Precipitants of oral cancer in India. Clin Cancer Investigation J 2012;1(3):111-113.

7. Shah G, Chaturvedi P, Vaishampayan S. Arecanut as an emerging etiology of oral cancers in India. Indian J Med Paediat Oncol 2012;33(2):71-79.

8. Neville BW, Damm DD, Allen CM, et al. Oral and maxillofacial pathology. 2nd ed. Philadelphia, PA: Saunders; 2002. p. 337-369.

9. Koss GL, Melamed MR, editors. Koss' Diagnostic cytology and its histopathologic bases. 5th ed. Lippincott Williams and Wilkins, Philadelphia. p. 11-12.

10. Sivapathasundharam B, Kalasagar M. Yet another article on exfoliative cytology. J Oral Maxillofac Pathol 2004;8(2):54-57.

11. Fontana L, Lasfargues G, Ughetto S, Rogier S, Masdieu E, Lafaure M, Cuvelier BA, Catilina P. The micronucleus assay in human exfoliated urothelial cells: application in a genotoxicity study of workers exposed to a mineral jelly containing sodium nitrite and N-phenyl-1-naphthylamine. Mutagenesis 2001;16(5):449-452.
12. Rosin MP. The use of the micronucleus test on exfoliated cells to identify anticlastogenic action in human: a biological marker for the efficacy of chemopreventive agents. Mutagen Res 1992;267(2):265-276.

13. Ramirez A, Saldanhan PH. Micronuclei investigation of alcoholic patients with oral carcinomas. Genetics Molecular Res 2002;1(3):246-260.

14. Fortin F, Anghel T, Brochu P, Lemieux N. Optimizing urothelial cell preparation for the human urinary micronucleus assay. Toxicol in vitro 2010;24(6):1821-1827.

15. Misra V, et al. Cytohistological study of urinary bladder neoplasms. Indian J Pathol Microbiol 2000;43(3):303-309.

16. Fenech M. Cytokinesis-block micronucleus cytome assay. Nature Protocols 2007;2(5):1084-1104.

17. Samanta S, Dey P. Micronucleus and its applications. Diagnostic Cytopathol 2012;40(1):84-90.

18. World Health Organization SEARO. Report on oral tobacco use and its implications in South-East Asia 2004.

19. Sarswathi TR, Ranganathan K, Shanmugam S, Ramesh S, Narsimhan DP, Gunaseelan R. Prevalence of oral lesions in relation to habits: cross-sectional study in South India. Indian J Dent Res 2006;17(3):121-125.

20. IARC. Monograph on evaluation of carcinogenic risk of chemicals to humans-tobacco habits other than smoking: betel-quid and areca-nut chewing. Lyon, France: IARC 1985; 37:141-202.

21. Zamani AG, Durakbasi-Dursun HG, Demirel S, Acar A. Evaluation of smoking genotoxicity in Turkish young adults. Indian J Human Genetics 2011;17(1):7-12. 